\title{
Monte Carlo algorithm for drift and diffusion of ions in anisotropic, non-homogeneous media
}

\author{
Goran Stojmenovik ${ }^{1}$, Filip Beunis ${ }^{2}$, Kristiaan $\mathrm{Neyts}^{2}$, Stefaan Vermael ${ }^{3}$, \\ Alwin R. M. Verschueren ${ }^{4}$ \\ ${ }^{1}$ Barco n.v., Noordlaan 5, B-8520, Kuurne, Belgium, goran.stojmenovik@barco.com \\ ${ }^{2}$ Liquid Crystals \& Photonics group, Elis Department, Ghent University, Sint- \\ Pietersnieuwstraat 41, B-9000 Gent, Belgium \\ ${ }^{3}$ Renson Ventilation n.v., Industriezone 2 Vijverdam - Maalbeekstraat 10, B-8790 \\ Waregem, Belgium \\ ${ }^{4}$ Philips Laboratories, Prof. Holstlaan 4, 5656 AA Eindhoven, The Netherlands
}

Keywords: ion transport, liquid crystals, Monte Carlo, anisotropic diffusion

A new Monte Carlo algorithm for ion transport in two-dimensional non-homogeneous anisotropic media with a barrier is reported. It is based on physical considerations of drift and diffusion in anisotropic media with or without an impermeable boundary. Non-homogeneities in the medium and electric field can be taken into account by averaging along the ion trajectory. The algorithm has been applied to the calculation of ion transport in liquid crystal displays and has been successfully compared with a finite difference program on a one-dimensional liquid crystal structure.

\section{Introduction}

When considering the drift and diffusion of charged particles (ions) in a free anisotropic medium in the presence of an electric field, the equation for the current density and the continuity equation yield the following, drift-diffusion equation:

$$
\pm \nabla[n(\mathbf{r}, t) \stackrel{\bar{\mu}}{\mathbf{E}}(\mathbf{r}, t)]-\nabla[\overline{\bar{D}} \nabla n(\mathbf{r}, t)]=-\frac{\partial n(\mathbf{r}, t)}{\partial t}
$$

where $n(\mathbf{r}, t)$ is the ion concentration as function of the position $\mathbf{r}$ and time $\mathrm{t}, \bar{\mu}$ is the ion mobility tensor, $\mathbf{E}(\mathbf{r}, t)$ is the electric field vector and $\overline{\bar{D}}$ is the diffusion tensor.

In case of one single particle, this equation can be solved in the principle coordinate system of the anisotropic medium, to yield solutions in terms of Gaussian probability functions for diffusion with the center of the distribution drifting according to product of the mobility tensor and electrical field vector $(d \mathbf{r}=\overline{\bar{\mu}} \mathbf{E} d t)$. This solution can be integrated using suitably small time steps to calculate the ion distribution after a given time.

However, if the anisotropic medium is inhomogeneous, there will be calculation errors because the drift displacement is linear with the time step, but the diffusion displacement is not. If we have an impermeable barrier, the ion motion is even more complicated. If the electric field presses the ions against the barrier, they will eventually form a steady state distribution, in which they bounce to and from a wall. The calculation errors in this case will cumulate, leading to a net ion motion in one direction along the barrier. We want to avoid this situation as our aim is to calculate precisely the ion motion in all three dimensions. 


\section{Model}

We have developed a Monte Carlo algorithm that calculates ion motion in the above mentioned case (anisotropic, inhomogeneous medium with an electric field and a barrier). The algorithm is split in 3 parts: 1) determination of the new ion position along the $z$-direction (perpendicular to the barrier); This is done by using statistical averages, i.e. solutions of the steady state equation in ideal cases ([1], [2], [3]); 2) calculation of the new ion position along the $x$ - and $y$-directions (parallel to the barrier) based on an analytical solution of the drift-diffusion equation in an arbitrary coordinate system, and 3) correction of the trajectory by averaging for the electric field and medium properties along the ion trajectory.

\section{Simulation results}

We have implemented the Monte Carlo algorithm in an existing 2-D simulation program for liquid crystals [4]. Using this program, we calculated the ion trajectory during two AC pulses through a TN liquid crystal. The same problem was calculated by using an already existing program - Glue ([5]) for 1-dimensional ion transport simulations. The calculated trajectories corresponded very well[6].

\section{Conclusions}

The algorithm we propose allows for calculation of the ion transport in anisotropic, non-homogeneous media with a boundary, such as liquid crystal cells. It can be implemented to calculate for example ion transport and image sticking properties in two-dimensional liquid crystal cells, like IPS, S-PVA or MVA.

\section{References}

[1] S. Vermael, et al., Proceedings of SPIE, 4759, 246, 2002

[2] S. Vermael, et al., Proceedings of Asia Display/IDW 2001, 33, 2001

[3] S. Vermael, PhD Thesis, Universiteit Gent 2003-2004 (in Dutch)

[4] F. Di Pasquale, et al., IEEE Journal of selected topics in quantum electronics, 2, 128, 1996

[5] C. Colpaert, A. De Meyere, B. Maximus, SID International Symposium Digest of Technical Papers XXVII, 301, 1996

[6] G. Stojmenovik, PhD Thesis, Universiteit Gent 2004/2005

Acknowledgments: This paper is part of a PhD thesis at Ghent University, Belgium. The author would like to acknowledge financial and scientific support by Philips Research, Eindhoven, The Netherlands. The ion transport research is also supported by the European SAMPA Network. 